\title{
Assessment of Eustachian Tube Function in Patients With Chronic Otitis Media With Effusion
}

\author{
Lingxue Zhang ${ }^{1}$, Shan $\mathrm{Liu}^{1}$, Li Xie ${ }^{1}$, Tejvansh Shenoy Imrit ${ }^{1}$, and Aiguo Liu ${ }^{1}$ \\ ${ }^{1}$ Tongji Hospital of Tongji Medical College of Huazhong University of Science and \\ Technology
}

May 4, 2020

\begin{abstract}
Objective: To explore the pathogenesis of Eustachian tube dysfunction (ETD) in patients with chronic otitis media with effusion (COME) using middle ear pressure-equilibration testing. Methods: Pressure equilibration testing of ET ventilation function was carried out in 72 ears which included 51 ears of COME patients with ventilation tube (COME group) and 21 ears of patients with traumatic tympanic perforation (TP group). According to the results of the test, these ears were further divided into four types: 1 . with both passive and active opening, 2. only with passive opening, 3. only with active opening, 4 . without passive and active opening. Results: $85.7 \%$ ears in the TP group had both active and passive ET function while $88.2 \%$ ears in the COME group had ETD. The average frequency of ET opening $(\mathrm{P}<0.001)$ and the occurrence rate of pressure rebound wave $(\mathrm{P}<0.001)$ was significantly lower in the COME group, while the mean residual pressure after several swallowing acts $(\mathrm{P}<0.001)$ and the average ET opening duration $(\mathrm{P}=0.004)$ was significantly higher in the COME group than in the TP group. Conclusions: Relatively higher residual pressure, lower effective opening frequency, disappearance of rebound wave and longer ET opening duration indicate poor ET ventilation function and an abnormal contraction pattern of peritubular muscles of the ET in patients with COME. Our results show that ETD is prevalent in most patients with COME with various causes. Therefore, appropriate treatment methods should be adopted to ensure prompt and adequate recovery. Keywords: Eustachian tube function,pressure-equilibration test,chronic otitis media with effusion
\end{abstract}

\section{Introduction}

Eustachian tube (ET) connects the middle ear (ME) cavity and nasopharynx and has three main physiological functions: ventilation of the ME, drainage and clearance of secretions produced within the ME and protection from nasopharyngeal sound pressure and secretions. ${ }^{1}$ The ET is usually closed under normal circumstances, opening happens either actively or passively. Passive opening occurs when the ET opens as a result of increased ME or nasopharyngeal pressure while active ET opening usually happens during swallowing. ${ }^{2}$ The $\mathrm{ME}$ is an air cavity which is highly susceptible to perturbations in atmospheric pressure and variations in middle ear pressure(MEP) most often occur due to passive diffusion of nitrogen between the ME and the nasopharynx. ${ }^{3}$ When the ventilatory function of the ET is impaired, normal gas transfer between the ME and nasopharynx is disrupted resulting in negative MEP and transudation of fluid from local capillaries. All this ultimately leads to otitis media with effusion (OME). In recent years, the incidence of diseases related to eustachian tube dysfunction(ETD), such as OME and adhesive otitis media has gradually increased. Some patients may require multiple tympanostomies or other surgical procedures because of chronic or recurrent OME (COME/ROME). To date, although the pathogenesis of ETD has not been completely elucidated, it has been suggested that ET ventilatory function could be closely related to the prognosis of COME. ${ }^{4}$ Therefore, accurate measurement of ET ventilatory function is essential to clarify the role of ETD in COME so as to formulate interventions and eventually, to be able to predict the efficacy of surgery.

A high-resolution digital manometer was used to perform the modified ME pressure-equilibration test ${ }^{5,6}$ on 
ears with perforated tympanic membranes and those with ventilation tube inserted(VTI). Details of the tests were recorded to help us find clues to the pathogenesis of ETD. Our primary aims were: 1 . to explore the roles of the two main components of ETD in the formation of COME, i.e., the resistance of ET opening and the contraction of the peritubular muscle, 2. to study the differences in magnitude and duration of ET opening between patients with COME and those with tympanic perforation (TP).

\section{Materials and Methods}

\section{Subjects}

We enrolled two groups of patients from January 2015 to January 2016. The TP group was composed of 20 patients(21 ears) with traumatic tympanic perforation (mean age, $39.6 \pm 13.8$ years; range, 14 to 65 years; male/female, 9/11), normal otoscopic findings prior to testing and no significant otologic history. This group was assumed to be representative of the population with normal ET function. The COME group consisted of 37 patients (51 ears) with chronic OME (mean age, $41.9 \pm 14.6$ years; range,12 to 64 years; male/female, 19/18) and a persistent type B tympanogram after conservative treatment for 3 months. These patients underwent tympanostomy tube placement and the test was carried out on the day following surgery (at this time the ET most closely resembled its pre-operative state). Patients with craniofacial abnormalities such as cleft palate, those with a history of radiotherapy following nasopharyngeal tumors and those with acute upper respiratory tract infection and otorrhea were excluded.

\section{Procedures and ETF testing Measurements}

Eustachian tube function (ETF) was measured in each ear using a modified pressure-equilibration test. An earplug containing two probes was introduced into the ear canal. One probe was connected to a constant flow pump at a speed of $25 \mathrm{ml} / \mathrm{min}$, the other probe was coupled to a high-resolution manometer and data of MEP was obtained from a computer which showed the MEP-time curve. The ME was inflated and positive pressure was applied. When the ET spontaneously opened, the pump was manually stopped and air was discharged through the ET until the latter closed passively. The pressure at which the ET opened passively was the opening pressure( $\mathrm{Po})$ and the pressure at which it closed passively was the closing pressure(Pc). We restricted the upper limit of positive pressure to $700 \mathrm{daPa}$ because some patients felt uncomfortable when the pressure exceeded the above level. If the ET remained closed when the pressure reached the upper limit, we defined it as negative passive opening function. The active opening function test was then carried out (Fig. 1), ${ }^{7}$ the ME was first inflated to $+200 \mathrm{daPa}$ following which the patient was instructed to swallow at least ten times. The residual pressure $(\mathrm{Pr})$ remaining in the $\mathrm{ME}$ after swallowing was recorded. The number of swallows and number of times the ET opened (judged by the appearance of the swallow waveform) were also noted down. Each time the ET opened, the MEP change was recorded as $\Delta \mathrm{P}$ and the duration of ET opening was recorded as $\Delta$ t (Fig. 2). Next, similar testing was carried out at a MEP of -200 daPa. To improve accuracy and misjudgment of ET opening, ET opening was only defined when $\Delta \mathrm{P}>3 \mathrm{daPa}$ and $\Delta \mathrm{t}<1.5 \mathrm{~s} .^{8}$ Patients were classified into four types according to the test results: 1 . with both active and passive ET opening function; 2. with only passive opening function; 3. with only active opening function; 4. without active or passive opening function.

Statistical analysis

Data obtained were statistically analyzed using SPSS version 17.0. Chi-square test, t-test and Mann-Whitney $\mathrm{U}$ test were used to analyse the date, and $\mathrm{P}<0.05$ was considered statistically significant.

\section{Results}

A total of 72 ears underwent our testing. These included 21 ears of TP patients and 51 ears of COME patients. There was no statistical difference in age and gender between the two groups (Table 1). Our results showed that $85.7 \%$ ears of TP patients had active and passive ET opening function while only $11.8 \%$ of COME ears had similar findings; $13.7 \%$ ears of COME patients and $4.8 \%$ ears of TP subjects had only passive opening function while $27.5 \%$ ears of COME patients and $9.5 \%$ ears of TP patients only had active opening function; $47.1 \%$ ears of COME patients did not demonstrate both active and passive opening function andnone ears 
of the TP subjects depicted this phenomenon(Table 2). There was a significant difference in both active and passive ventilation function between the two groups $(\mathrm{P}<0.001) .85 .7 \%$ ears in the TP group had both active and passive ET function while $88.2 \%$ ears in the COME group had ETD.

We found 90.5\% (19/21) ears with TP whose ETs could be passively opened. The ranges of Po and Pc were each $215^{\sim} 480 \mathrm{daPa}$ and $25^{\sim} 160 \mathrm{daPa}$ and the mean values were $305.1 \pm 41.6 \mathrm{daPa}$ and $82.1 \pm 37.2 \mathrm{daPa}$ respectively. In the COME ears, only $25.5 \%(13 / 51)$ ETs had passive opening function; the ranges of Po and $\mathrm{Pc}$ were $230 \sim 491 \mathrm{daPa}$ and $30^{\sim} 105 \mathrm{daPa}$, with a mean of $292.5 \pm 59.6 \mathrm{daPa}$ and $70.5 \pm 20.7 \mathrm{daPa}$ respectively. However, no significant differences in $\mathrm{Po}(\mathrm{P}=0.49)$ and $\mathrm{Pc}(\mathrm{P}=0.27)$ were found in our test (Table 3$)$.

In the TP group, 95.2\% (20/21) ears had active ET opening under positive MEP, and 85.7\% (18/21) ETs could open actively during the negative phase. The ranges of Pr under positive MEP and negative MEP were $0 \sim 50 \mathrm{daPa}$ and $-103^{\sim} 0 \mathrm{daPa}$. In those patients with active ET opening function, we observed that almost every swallow could trigger an ET opening (Fig. 3)with the magnitude of each ET opening $(\Delta \mathrm{P})$ ranging from 8 to $85 \mathrm{daPa}$ and the mean ET openings during 10 swallows was 9.1. The waveform after each opening had very good consistent shape and the mean duration of the ET opening was $0.50 \pm 0.13 \mathrm{~s}$. While recording the MEP-time curve, a rebound wave (Fig. 2, Fig. 3) could be seen at the tail of the waveform in 90\% (19/21) ears. In contrast, $39.2 \%(20 / 51)$ ears from the COME group had active ET opening under positiveMEP and only $7.8 \%$ (4/51) ears had passive opening under negativeMEP.The ranges of Pr under positive MEP and negative MEP were $35^{\sim} 180 \mathrm{daPa}$ and $-192^{\sim}-98$ daPa respectively. The mean Pr under positive MEP was much higher in the COME group $(99.7 \pm 46.1 \mathrm{daPa})$ than that in the TP group $(16.9 \pm 16.8 \mathrm{daPa})(\mathrm{P}<0.001)$. In our tests, we observed that the features of the swallowing waves in COME group performed differently with the TP group (Fig. 4). Some patients under each swallow could trigger an active ET opening but the magnitude of each opening was very small $(\Delta \mathrm{P}<8 \mathrm{daPa})$.Among the patients with ET active opening function, not every swallow resulted in effective opening of the ETor the ET even remained unopened, and we found the average ET openings in 10 swallows was 3.8 in COME groupwhich was much less in comparison to those patients in the TP group $(\mathrm{P}<0.001)$; in addition, the rebound wave could only be observed in $20 \%$ ears in the COME group which was again significantly less than that in the TP group $(\mathrm{P}<0.001)$. The mean duration of the ET opening was $0.63 \pm 0.13 \mathrm{~s}$, which was slightly longer than that in the TP group $(\mathrm{P}=0.004)$ (Table 3).

\section{Discussion}

The ventilation function of the ET includes active and passive openingwhich are key for the success of ME surgeries such as tympanoplasty and myringotomy with VTI. ${ }^{4,9,10}$ Its dysfunction can lead to many ME diseases including COME, atelectasis and cholesteatoma formation. ${ }^{2,11,12}$ Nasopharyngeal obstructions such as adenoid hypertrophy, tumor compression and intratubular mucus retention mainly cause passive opening dysfunction while impaired contraction of the peritubular muscles, i.e., tensor veli palatini (TVP) and levator veli palatini (LVP) mostly leads to active opening dysfunction. ${ }^{13}$ The presence of passive opening is a good indication that the ET is free of obstruction while active opening indicates that the ET can be actively opened by effective contraction of the peritubular muscles.

In this study, most ears in the TP group had both active and passive ET ventilation function. Only $9.5 \%(2 / 21)$ ears failed in passive ET opening, 4.7\%(1/21) ears could not balance the positive MEP and $14.3 \%(3 / 21)$ could not balance the negative MEP. Elner and colleagues ${ }^{14}$ explored the normal ETF in 102 healthy subjects and found that the ET was forced to open passively in all tested subjects, $5 \%$ of subjects could not equilibrate positive MEP and 7\% could not equilibrate negative MEP. The results were comparable to ours and indicated that a few normal subjects with no ear diseases also had impaired ETF. We speculated these subjects were more susceptible to otitis media compared to those with good ETFand were thus more likely to develop ME diseases such as OME in the presence of infection, immunological and other induced factors. ${ }^{15}$

We evaluated both the active and passive ET ventilation function of 21 ears in the TP group and 51 ears in the COME group and classified those ears into four categories as mentioned before(Table 2). We found $88.2 \%$ ears in the COME group had either active or passive ventilation dysfunction of ET. The possible 
reasons for the ETD included: 1.Decreased contractility of the peritubular muscles in cases where ET could undergo only passive opening, with testing showing normal ET opening resistance; 2.Increased ET opening resistance with normal or even enhanced peritubular muscle function in cases where ET could only undergo active opening; 3. In cases with both active and passive opening failure, there was very high ET opening resistance which probably exceeded the maximum contractility of the peritubular muscles. So it is necessary to choose appropriate treatment according to various causes of poor ventilation of the ET. Rehabilitation of the peritubular muscle should be emphasized in the treatment of muscular abnormalities; and drugs to reduce mucosal edema, to promote mucus excretion or the use of surfactants are the possible treatment options to reduce increased ET opening resistance caused by mucosal inflammation.

Po is related to the opening resistance of the ET (mucosal factors, surface tension, viscosity of secretions, etc.) ${ }^{16}$ When the resistance is increased, a higher positive MEP is needed to open the ET. If the resistance is greater than the maximum pressure that can be applied to force the ET to open passively, the tube will not be opened. Pc is assumed to represent the periluminal forces (pressure of the cartilage and other surrounding tissues, etc.) and is related to the protective function of the ET. ${ }^{17}$ Our results showed that $90.5 \%$ ears in the TP group had passive ET opening with a Po and Pc range of $215^{\sim} 480$ dapa and 25 160daPa respectively. This was comparable to the results reported by Takahashi. ${ }^{11}$ In contrast, only $25.5 \%$ of ears in the COME group had passive ET opening with their Po and Pc range close to that of the TP group. There was a significant difference in the proportion of the two groups $(\mathrm{P}<0.05)$. This indicated that a majority of COME patients had increased ET opening resistance which resulted in the inability to effectively open the ET.

ET is an elastic tubular structure composed of a mucosacovered lumen, cartilage, peritubular muscles and soft tissues. The opening of the ET begins at the cartilaginous anterior one-third segment of the ET lying at the nasopharyngeal orifice. Opening is triggered by contraction of the LVP which then spreads to the isthmus by contraction of TVP to open the posterior $2 / 3$ of the tube. Tubal closure is brought about by the elastic recoil of the tube and peritubular tissues and relaxation of LVP and TVP although the direction followed is opposite to that seen on opening, i.e., closure begins from the tympanic end of the ET and relaxation of the TVP is followed by that of the LVP. ${ }^{18,19}$ This mechanism of tubal opening is able to explain the formation of the rebound wave we observed in our study. The appearance of this wave is assumed to reflect the extent of opening and the speed of closing. While no relevant literature has reported this phenomenon so far, in our study, we observed the rebound wave in most TP patients and the appearance of this rebound wave during swallowing was significantly lower in the COME group in comparison to the TP group $(\mathrm{P}<0.001$, Table3). Thus, we could speculate that the contraction pattern of the peritubular muscles in COME patients might be different from normal and healthy subjects. It is a known fact that owing to chronic inflammation of the ME and ET, COME patients have associated mucosal edema and hyperplasia and narrowing of the ET lumen. As a result, the peritubular muscles require more effort to open the tube which in turn leads to a smaller opening. Moreover, since the contractile effort is increased, the peritubular muscles would also have decreased velocity of contraction and relaxation. Both decreased extent of tubal opening and decelerated closing thus leaded to the disappearance of the rebound wave.

The level of Pr after swallowing is an indicator of the active pressure equilibrating ability of the ET and many researchers have graded the ETF according to it. ${ }^{14,20}$ The higher Pr after several swallows is usually related to the poor active opening of ET. In our study, we observed that almost every swallow could trigger an ET opening in the TP group with active ET opening function with post-swallow Pr ranging from 0 to 50 daPa under positive MEP and -103 to 0 daPa under negative MEP respectively. In the COME group, not every swallow could open the ET effectively; only 2 ears had a Pr less than 50 daPa when given a positive MEP and most ears could not open actively under negative MEP. The mean positive-Pr was higher in the COME group compared to the TP group $(\mathrm{P}<0.001$, Table3). Active opening of the ET is powered by contraction of the peritubular muscles (TVP, LVP and salpingopharyngeus) during swallowing or yawning. ${ }^{18,21}$ We speculated the higher Pr in COME patients could be related to the low frequency of ET opening during 10 consecutive swallows which was 3.8 times compared to9.1 times in the TP group. This phenomenon was also observed by Van Heerbeck and colleagues, ${ }^{22}$ they found the frequency of ET opening during 10 swallows dropped from 8.4 times to 2.7 times after inducing ET dysfunction in healthy subjects. One explanation for this phenomenon 
is that there is usually more secretions in a diseased ET lumen which accounts for the increased opening resistance. Consequently, with greater ET opening resistance, more muscular force would be required to open the ET. Additionally, after each swallow, the opening resistance may vary due to the changeable distribution of secretions in the lumen; the ET would only open actively under a circumstance where the resistance is relatively smaller. ${ }^{23}$ Another explanation is that the peritubular muscles suffer increased fatigue and muscle contraction abnormalities (e.g., fasciculations, hypercontractility and limited contractility) during successive deglutition which may cause ET to open not at all or only partially. ${ }^{24}$

The mean ET opening duration in the TP group was $0.50 \pm 0.13 \mathrm{~s}$ in our study. Mondain ${ }^{25}$ reported a mean duration of $0.43 \pm 0.18 \mathrm{~s}$ in 120 normal ears using sonotubometry which closely resembles our results. Gaihede ${ }^{8}$ reported a mean ET opening duration of $0.34 \mathrm{~s}$ in their pressure-equilibration test, however, there were only 4 healthy subjects included in hisstudy. We observed a significantly longer duration of ET opening in the COME group than in the TP group $\left(\mathrm{P}=0.004\right.$,Table 3), Poe and Alper ${ }^{26,27}$ also observed a longer duration of opening in patients with ETD compared to normal subjects using slow-motion endoscopy. The reason for this phenomenon has not been fully understood up till now though decreased contraction and relaxation speed of the peritubular muscles caused by increased afterload (ET opening resistance) could be the cause.

\section{Conclusion}

The relatively higher Pr, lower effective opening frequency, disappearance of rebound wave and longer ET opening duration indicate poor ET ventilation function and abnormal contraction pattern of peritubular muscles of ET in COME patients.Most patients with COME have either active or passive ET ventilation dysfunction due to different causes. Etiological therapy should be adopted to ensure prompt and adequate recovery.

\section{References}

1 Sadé J, Ar A. Middle ear and auditory tube: middle ear clearance, gas exchange, and pressureregulation. Otolaryngology-Head and Neck Surgery 1997;116:499-524.

2 Cantekin EI, Saez CA, Bluestone CD, Bern SA. Airflow through the eustachian tube. Ann Otol Rhinol Laryngol 1979;88:603-12.

3 Ostfeld EJ, Silberberg A. Transient pressure changes in the middle ear. Arch Otolaryngol Head Neck Surg 1991;117:1390-4.

4 Mandel EM, Swarts JD, Casselbrant ML, et al. Eustachian tube function as a predictor of the recurrence of middle ear effusion in children.Laryngoscope 2013;123:2285-90.

5 Bluestone CD, Cantekin EI. Current clinical methods, indications and interpretation of eustachian tube function tests. Ann Otol Rhinol Laryngol 1981;90:552-62.

6 Cantekin EI. Eustachian tube function in children with tympanostomy tubes. Auris Nasus Larynx 1985;12 Suppl 1:S46-8.

7 van Heerbeek N, Ingels KJ, Snik AF, Zielhuis GA. Reliability of manometric eustachian tube function tests in children. Otol Neurotol 2001;22:183-7.

8 Gaihede M, Padurariu S, Jacobsen H, De Greef D, Dirckx, JJ. Eustachian tube pressure equilibration. Temporal analysis of pressure changes based on direct physiological recordings with an intact tympanic membrane.Hear Res 2013;301:53-9.

9 Takahashi H, Sato H, Nakamura H, Naito Y, Umeki H. Correlation between middle-ear pressure-regulation functions and outcome of type-I tympanoplasty. Auris Nasus Larynx 2007;34:173-6.

10 Choi SH, Han JH, Chung JW. Pre-operative Evaluation of Eustachian Tube Function Using a Modified Pressure Equilibration Test is Predictive of Good Postoperative Hearing and Middle Ear Aeration in Type 1 Tympanoplasty Patients. Clinical and Experimental Otorhinolaryngology 2009;2:61-5. 
11 Takahashi H, Hayashi M, Sato H, HonjoI. Primary deficits in eustachian tube function in patients with otitis media with effusion. Arch Otolaryngol Head Neck Surg 1989;115:581-4.

12 Casselbrant ML, Mandel EM, Seroky JT, Swarts JD, Doyle WJ. A pilot study of the ability of the forced response test to discriminate between 3-year-old children with chronic otitis media with effusion or with recurrent acute otitis media. Acta Oto-Laryngologica 2011;131:1150-4.

13 Doyle WJ, Swarts JD, Banks J, Casselbrant ML, MandelEM. Sensitivity and Specificity of Eustachian Tube Function Tests in Adults. JAMA Otolaryngol Head Neck Surg 2013;139:719-27.

14Elner A, Ingelstedt S, Ivarsson A.The normal function of the Eustachian tube:A study of 102 cases. Acta Otolaryngol (Stockh) 1971;72:320-8.

15Buchman CA, Doyle WJ, Skoner D, Fireman P, GwaltneyJM. Otologic manifestations of experimental rhinovirus infection. Laryngoscope 1994;104:1295-9.

16 Cantekin EI, Bluestone CD, Parkin LP. Eustachian tube ventilatory function in children. Ann Otol Rhinol Laryngol 1976;85:171-7.

17 Stenström C, Bylander-Groth A, Ingvarsson L. Eustachian tube function in otitis-prone and healthy children. Int J Pediatr Otorhinolaryngol 1991;21:127-38.

18 Lin C, Li Y, Lin X, Zhang S, Tang A, Nong D. Cross-platform data integration of paratubal electromyography and sonotubometry for eustachian tube: a pilot study. Acta Otolaryngol 2016;136:1130-6.

19 Ishijima K, Sando I, Balaban CD, Miura M, Takasaki K. Functional anatomy of levator veli palatini muscle and tensor veli palatini muscle in association with eustachian tube cartilage. Ann Otol Rhinol Laryngol 2002;111:530-6.

20 van Heerbeek N, Ingels K, Snik AF, Zielhuis GA. Eustachian tube function in children after insertion of ventilation tubes. Ann Otol Rhinol Laryngol 2001;110:1141-6.

21 Okada R, Muro S, Eguchi K, et al. The extended bundle of the tensor veli palatini: Anatomic consideration of the dilating mechanism of the Eustachian tube.Auris Nasus Larynx 2018;45:265-72.

22 van Heerbeek N, van der Avoort SJ, Zielhuis GA, CremersCW. Sonotubometry: a useful tool to measure intra-individual changes in eustachian tube ventilatory function. Arch Otolaryngol Head Neck Surg 2007;133:763-6.

23 Poe DS, Abou-Halawa A, Abdel-Razek O. Analysis of the dysfunctional eustachian tube by video endoscopy. Otol Neurotol 2001;22:590-5.

24 Mathew GA, Kuruvilla G, Job A. Dynamic slow motion video endoscopy in eustachian tube assessment . Am J Otolaryngol 2007;28:91-7.

25 Mondain M,Vidal D,Bouhanna S,Uziel A.Monitoring eustachian tube opening: preliminary results in normal subjects.Laryngoscope 1997;107:1414-9.

26 Poe DS, Pyykko I. Measurements of Eustachian tube dilation by video endoscopy. Otol Neurotol 2011;32:794-8.

27 Alper CM, Teixeira MS, Swarts JD, Doyle WJ. Quantitative Description of Eustachian Tube Movements During Swallowing as Visualized by Transnasal Videoendoscopy. JAMA Otolaryngol Head Neck Surg $2015 ; 141: 160-8$.

\section{Hosted file}

Table.docx available at https ://authorea.com/users/317485/articles/447563-assessment-of-eustachiantube-function-in-patients-with-chronic-otitis-media-with-effusion 

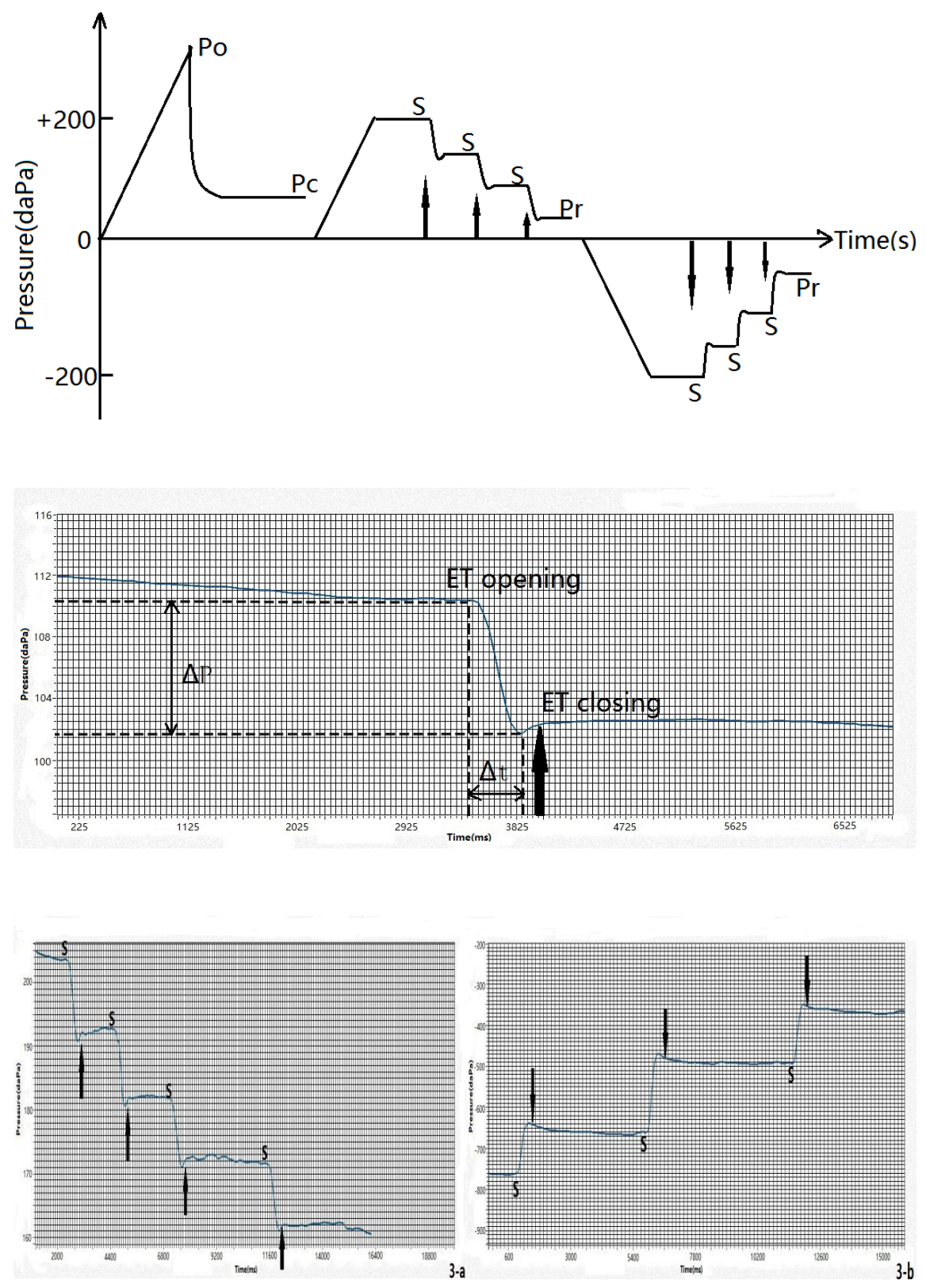

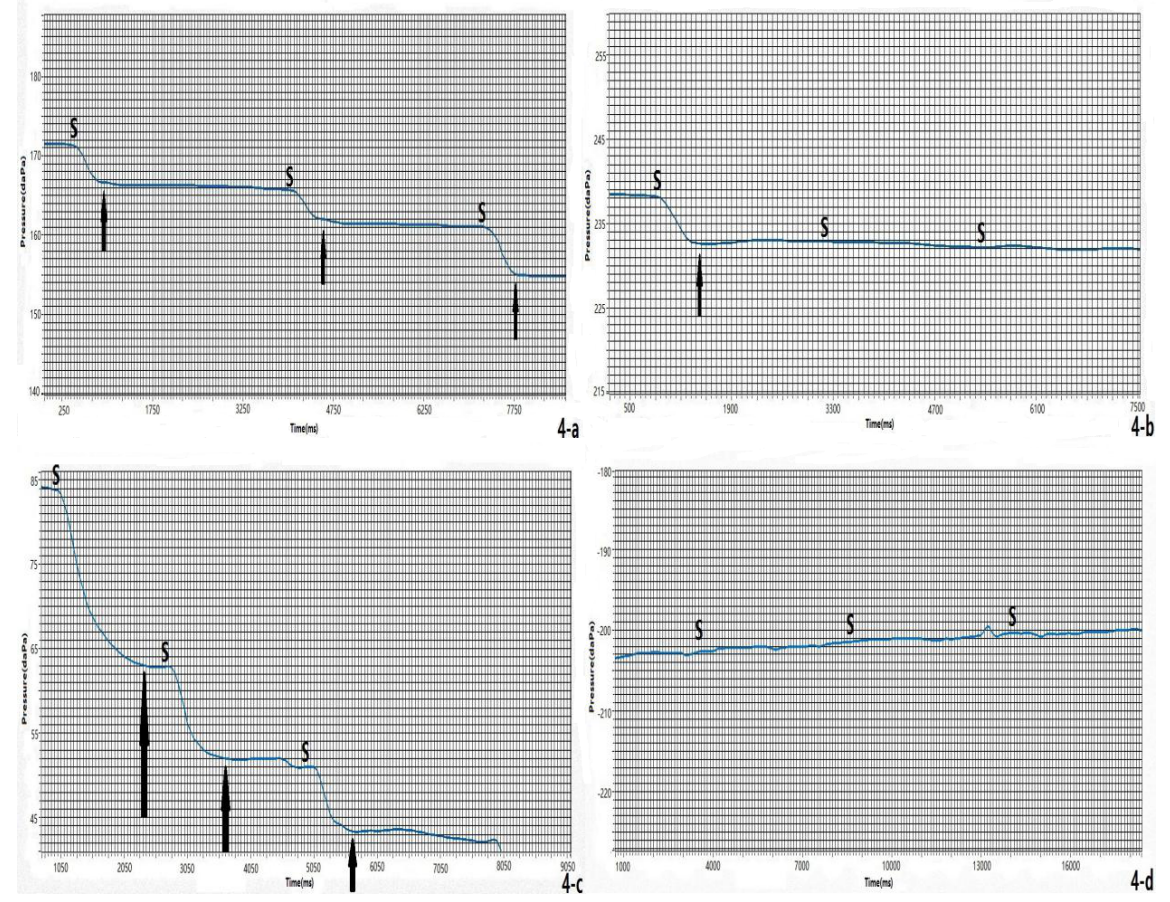